\title{
Antiplatelet therapy - a summary for the general physicians
}

\author{
Author: Jecko Thachil ${ }^{\mathrm{A}}$
}

Platelets play a very important role in physiological haemostasis and thrombus formation. Platelet aggregation is the key pathophysiological factor in the development of arterial ischaemic events, including coronary artery disease, cerebrovascular accidents and peripheral arterial disease. As such, antiplatelet therapy plays a very important role in preventing recurrent events in the individuals who are affected by one of these conditions. Until recently, the repertoire of antiplatelet therapy was limited to aspirin and clopidogrel. However, this landscape has changed dramatically with the advent of newer and more potent agents, prasugrel and ticagrelor and also the glycoprotein IIb/IIIa antagonists. This armamentarium is likely to expand further with the advent of protease-activated receptor- 1 antagonists and the intravenous cangrelor. This review summarises the different agents available and some practical considerations for their use from a general physician's perspective.

KEYWORDS: Platelets, antiplatelet aspirin, clopidogrel, platelets, prasugrel, ticagrelor

\section{Introduction}

Platelets play a very important role in physiological haemostasis and thrombus formation. Platelet aggregation is the key pathophysiological factor in the development of arterial ischaemic events including coronary artery disease, cerebrovascular accidents and peripheral arterial disease. As such, antiplatelet therapy plays a very important role in preventing recurrent events in the individuals who are affected by one of these conditions. Until recently, the repertoire of antiplatelet therapy was limited to aspirin and clopidogrel. However, this landscape has changed dramatically with the advent of newer and more potent agents, prasugrel and ticagrelor and also the glycoprotein IIb/ IIIa antagonists. This armamentarium is likely to expand further with the advent of protease-activated receptor-1 (PAR-1) antagonists and the intravenous cangrelor. This

Author: ${ }^{A}$ consultant haematologist, Department of Haematology, Central Manchester University Hospitals NHS Foundation Trust, Manchester, UK. review summarises the different agents available and some practical considerations for their use from a general physician's perspective.

For the purpose of this review, the antiplatelet drugs are categorised into three based on their arrival into the market the past, present and future.

\section{The past}

Aspirin works as an antiplatelet agent by irreversibly blocking the enzyme cyclooxygenase-1 (COX-1) inside the platelets. ${ }^{1}$ This enzyme is necessary to generate thromboxane A2, a potent platelet activator from arachidonic acid. ${ }^{1}$ Aspirin also inhibits COX-2 which explains part of its anti-inflammatory properties, although several other mechanisms including reduced prostaglandin generation and those involving the immune cells and cytokines have also been proposed. The gastrointestinal side effects of aspirin are caused by COX-1 inhibition. The maximal antithrombotic effect of aspirin is achieved with a daily dose of 75-100 mg with no further benefits from increasing the dose, although the risk of bleeding increases with the dose. ${ }^{2}$ Since aspirin selectively targets the COX pathway, platelet activation can continue through other pathways, necessitating the need for additional agents like clopidogrel for full antithrombotic function.

Clopidogrel belongs to a class of drugs which are classed under adenosine diphosphate (ADP) antagonists. ${ }^{3} \mathrm{ADP}$ is an important participant in the platelet aggregation process by interacting with two puringeric receptors on platelets called $\mathrm{P}_{2} \mathrm{Y}_{1}$ and $\mathrm{P}_{2} \mathrm{Y}_{12}$ with the latter having more specific tissue distribution. ${ }^{3}$ The first $\mathrm{P} 2 \mathrm{Y}_{12}$-receptor inhibitor approved for use was ticlopidine, however it was associated with unfavourable haematological effects. Clopidogrel is a secondgeneration $\mathrm{P}_{2} \mathrm{Y}_{12}$ blocker which requires conversion to an active metabolite for its antiplatelet effect. ${ }^{4}$ The need for active metabolite formation may cause a delay on the antiplatelet properties necessitating a loading dose to achieve maximal platelet inhibition. ${ }^{4}$ Clopidogrel is metabolised to its active metabolite partly by the liver enzyme CYP2C19. As such the use of concomitant drugs which will inhibit this enzyme should be avoided (eg omeprazole, fluoxetine, fluconazole and carbamazepine). ${ }^{4}$ The genetic polymorphisms of CYP2C19 recently described also impact on the availability, or delayed response to the active metabolite of the drugs metabolized by this enzyme. See Table 1 for pharmacological characteristics. 


\begin{tabular}{|c|c|c|c|}
\hline & Clopidogrel & Prasugrel & Ticagrelor \\
\hline $\mathrm{P} 2 \mathrm{Y}_{12}$ binding & Irreversible & Irreversible & Reversible \\
\hline Indication & $\begin{array}{l}\text { ACS treated medically or with PCI, ischaemic } \\
\text { stroke, peripheral artery disease }\end{array}$ & ACS post $\mathrm{PCI}$ & ACS \\
\hline \multirow[t]{2}{*}{ Dose } & $\begin{array}{l}300-600 \text { mg loading } \\
75 \text { mg maintenance }\end{array}$ & $\begin{array}{l}60 \text { mg loading dose, then } \\
10 \text { mg/day }\end{array}$ & $\begin{array}{l}180 \mathrm{mg} \text { loading dose, then } \\
90 \mathrm{mg} \text { bd }\end{array}$ \\
\hline & & $(5 \mathrm{mg}$ if $<60 \mathrm{~kg})$ & \\
\hline Prodrug & Yes; needs hepatic metabolism & Yes; needs hepatic metabolism & No \\
\hline Onset of action & $2-8$ hours & 30 min -4 hours & 30 min -4 hours \\
\hline Elimination time & $7-10$ days & $7-10$ days & $3-5$ days \\
\hline CYP metabolism & CYP2C19 & CYP2C19 & CYP3A4/5 \\
\hline
\end{tabular}

Dipyridamole was introduced in the 1960s as a coronary vasodilator. Since it was shown to inhibit the uptake of adenosine, a potent platelet aggregator, its antiplatelet role was further explored. ${ }^{5}$ It mechanisms of action are the inhibition of phosphodiesterase (PDE) and red cell-mediated uptake of adenosine, both of which lead to inhibition of platelet aggregation. ${ }^{5}$ Oral ingestion of dipyridamole requires low $\mathrm{pH}$ for absorption (may cause problems with concomitant antacid therapies). ${ }^{6}$ Extended release preparation of this drug is now favoured as an antiplatelet agent in the prevention of ischaemic stroke in combination with aspirin. ${ }^{7}$ Due to its vasodilating properties, dipyridamole can cause hypotension (careful with antihypertensives) and worsening of coronary heart disease. ${ }^{5,6}$ It can also aggravate myasthenia gravis by interacting with cholinesterase inhibitors. ${ }^{5,6}$

Cilostazol acts by selectively inhibiting PDE type III and was first approved for the treatment of intermittent claudication. ${ }^{8}$ Although it has antiplatelet properties, it still needs to be given in combination with another agent like aspirin or clopidogrel in patients with intermittent claudication. The American College of Chest Physicians guidelines recommend addition of cilostazol to aspirin or clopidogrel for patients who have refractory claudication despite exercise therapy and smoking cessation. ${ }^{9}$ In Asian countries, this drug has been used in addition to dual antiplatelet agents in patients receiving coronary stents and as an alternative to aspirin in patients with ischaemic stroke (less haemorrhagic risk compared to aspirin). ${ }^{8,10}$ The most common reported side effects of cilostazol are headache and diarrhoea.

\section{The present}

The prevalence of poor response to clopidogrel led to the development of the $\mathrm{P}_{2} \mathrm{Y}_{12}$-receptor blockers, prasugrel and ticagrelor. See Table 1 for pharmacological characteristics.

Prasugrel after oral intake also needs hepatic metabolism to generate its active metabolite, although, in comparison with clopidogrel this is more efficient, resulting in higher bioavailability, faster onset of action and most importantly, more potent antiplatelet effect than its predecessor., ${ }^{4,10} \mathrm{~A}$ loading dose of $60 \mathrm{mg}$ achieves platelet inhibition beginning at 30 minutes and maximal at $2-4 \mathrm{~h}$ in patients with stable coronary artery disease (delayed in acute myocardial infarction probably due to slower gastrointestinal absorption). ${ }^{10}$ The clinical efficacy of prasugrel was investigated in TRITON-TIMI 38 with with advantages over clopidogrel clearly notable in patients with ST elevation myocardial infarction (events $9.7 \%$ for clopidogrel vs $7.4 \%$ for prasugrel) and those with recurrent events, although a clear advantage was not observed in older individuals ( $\geq 75$ years) and those weighing less than $60 \mathrm{~kg}$ (dose reduction to $5 \mathrm{mg}$ maintenance advised). ${ }^{12,13}$ It had negative impact in those with a history of stroke or transient ischaemic attack.

Ticagrelor is another orally active $\mathrm{P}_{2} \mathrm{Y}_{12}$-receptor blocker but unlike clopidogrel and prasugrel does not require metabolic activation and reversibly inhibits the receptor., ${ }^{4,10,14}$ The previously mentioned drugs are irreversible inhibitors, which because platelets are anucleate, mean that they are inhibited for the duration of their lifespan in the circulation; but the effect of the drug diminishes as new platelets are formed. Administered twice daily, ticagrelor, similar to prasugrel, has a quick onset of action, is more potent than clopidogrel but has also got the advantage of faster offset of action. ${ }^{4,10}$ It also inhibits cellular adenosine uptake which can lead to effects like the sensation of dyspnoea. Inhibition of adenosine reuptake is also thought to contribute to anti-inflammatory and antiplatelet effects of ticagrelor. ${ }^{32}$ Based on the phase-III PLATO trial, ticagrelor is approved for the treatment and prevention of thrombotic events in patients with acute coronary syndrome (ACS), irrespective of whether invasive or non-invasive strategy has been chosen although not in patients with stable disease. ${ }^{14,15}$ Since it can precipitate arrhythmias, ticagrelor should not be used in patients with bradycardia, sick sinus syndrome or atrioventricular block. ${ }^{4,10,16}$ Also, patients receiving drugs metabolised by hepatic CYP3A4 and CYP3A5 enzymes should avoid this agent (eg clarithromycin, digoxin, simvastatin). ${ }^{16}$ It is also better avoided in patients with asthma or chronic obstructive pulmonary disease and also in those with a history of hyperuricaemia, because it can increase uric acid levels ${ }^{4,16}$ Creatinine levels may increase after initiation with ticagrelor. ${ }^{16}$ Glycoprotein IIb/IIIa is the receptor involved in platelet aggregation mediated predominantly by fibrinogen. There are three parenteral glycoprotein IIb/IIIa inhibitors (GPI) currently available: abciximab, eptifibatide and tirofiban. ${ }^{17}$ The latter 
two have a shorter half-life, and are renally excreted, while abciximab has a higher affinity for the receptor, and does not require dose adjustments in renal impairment. ${ }^{18}$ With the availability of potent antiplatelet agents in oral formulation, the use of GPI is currently restricted to patients with unstable angina who require percutaneous coronary intervention (PCI) and cannot be pretreated with a P2Y12 blocker. ${ }^{18}$ However, because of the fast onset of action and relatively quick elimination (approximately 4 hours), GPIs are increasingly being considered as a 'bridging' strategy for patients on dual antiplatelet therapy needing surgical procedures. ${ }^{18,19}$ In the peri-procedure setting, discontinuation of antiplatelet therapy for a considerable period can be associated with an increase in thrombosis. Randomised controlled trials are underway in this area.

\section{The future}

All three $\mathrm{P} 2 \mathrm{Y}_{12}$-receptor blockers being orally administered agents have the disadvantage that they cannot be given in unconscious or intubated patients or those with haemodynamic compromise and also if the patient has repeated vomiting. Cangrelor is an intravenous formulation which reversibly binds to the $\mathrm{P} 2 \mathrm{Y}_{12}$ receptor and reaches effective concentrations within minutes. ${ }^{4,10,20}$ It also has the advantage of stable pharmacokinetics, a very short half-life (5 minutes) - meaning rapid offset of action, and pre-treatment platelet aggregation levels achieved within 30-60 minutes. ${ }^{4,10}$ Possible use of cangrelor will be as a bridging strategy in patients with ACS who may need an urgent coronary artery bypass graft surgery. ${ }^{21}$

Thrombin at sites of vascular injury recruits platelets into forming a haemostatic plug. This process is mediated by receptors known as protease-activated receptors (PARs). Vorapaxar is an antagonist of PAR and has been approved for use in the reduction of thrombotic events in patients with a history of myocardial infarction or peripheral arterial disease, in combination with standard antiplatelet therapy. ${ }^{10,22}$ Its use is contraindicated in patients with a history of stroke, transient ischaemic attack or intracranial haemorrhage because of the increased risk of major bleeding. ${ }^{4}$

\section{Indications for antiplatelet therapy}

The indications for drugs with antiplatelet function as recommended by the National Institute for Health and Care Excellence and the American Heart Association guidelines are listed below. ${ }^{16,23}$

\section{Acute coronary syndrome}

> All patients should receive treatment with long-term with low-dose aspirin (75 mg daily). If intolerant of aspirin, clopidogrel (75 mg daily) is an alternative.

$>$ For patients with definite or likely non ST elevation ACS in whom an initial invasive or ischemia-guided strategy is selected: aspirin with a $\mathrm{P}_{2} \mathrm{Y}_{12}$ inhibitor; clopidogrel 300 or $600 \mathrm{mg}$ or ticagrelor $180 \mathrm{mg}$.

$>$ For non ST segment elevation ACS patients undergoing PCI with stenting: aspirin with a $\mathrm{P}_{2} \mathrm{Y}_{12}$ inhibitor, clopidogrel 300 or $600 \mathrm{mg}$ or ticagrelor $180 \mathrm{mg}$ or prasugrel $60 \mathrm{mg}$.

$>$ Maintenance is with aspirin $75 \mathrm{mg} /$ day continued indefinitely, plus a $\mathrm{P}_{2} \mathrm{Y}_{12}$ inhibitor for up to 12 months:

\section{Box 1. Which $P 2 Y_{12}$ inhibitor to choose. ${ }^{6}$}

Choose:

clopidogrel, when cost is an issue, there is a high risk of bleeding, in patients who have received thrombolysis, or in patients who need long-term oral anticoagulation

ticagrelor (in preference to clopidogrel), in patients managed by an ischemia-guided or invasively treated patients

prasugrel (in preference to clopidogrel), in patients undergoing PCI.

Avoid:

clopidogrel, if CYP2C19*2 polymorphisms present, and in those who need to take PPIs, especially omeprazole or esomeprazole

ticagrelor, if patients are receiving concomitant drugs which are strong inhibitors of CYP3A4

prasugrel, if patients had a history of stroke or TIA, age $>75$ years and body weight $<60 \mathrm{~kg}$.

$\mathrm{PCI}=$ percutaneous coronary intervention; PPIs = proton-pump inhibitors; $\mathrm{TIA}=$ transient ischaemic attack,

clopidogrel $75 \mathrm{mg} /$ day or ticagrelor $90 \mathrm{mg}$ twice daily (or PCI with stent prasugrel $10 \mathrm{mg} /$ day).

Box 1 gives a simple guide for the appropriate $\mathrm{P}_{2} \mathrm{Y}_{12}$ inhibitor to choose in each setting.

\section{Ischaemic stroke and transient ischaemic attack}

> Clopidogrel (75 mg daily) is the preferred antiplatelet. If intolerant of clopidogrel, aspirin in combination with modified-release dipyridamole (200 mg twice a day).

\section{Peripheral arterial disease}

> Clopidogrel $75 \mathrm{mg}$ daily is the preferred antiplatelet, if intolerant, aspirin or modified-release dipyridamole alone.

\section{For primary prevention in cardiovascular disease}

There has been interest in the use of aspirin for the primary prevention of cardiovascular disease based on the mortality reduction observed only if taken for over 10 years (but with the increased risk of bleeding). ${ }^{24}$ It may thus only be prescribed to the patients who are well informed of their cardiovascular risk status and understand the need to take the drug long-term for small benefits (unlicensed use). ${ }^{16}$ The Framingham risk score that predicts the 10 -year risk of developing a cardiovascular event may be used in this setting to provide the patient with an estimate of event risk. The British Heart Society recommends restriction of aspirin to patients over 50 years with 10 -year cardiovascular risk of at least $20 \%$ after blood pressure has been adequately controlled and with no contraindication to aspirin use. $^{16}$

\section{Bleeding risk with antiplatelet drugs}

Gastrointestinal bleeding is the commonest adverse event associated with any antiplatelet agent. Peptic ulcers are 
the commonest cause and often occur without symptoms of dyspepsia. The main risk factors for this complication include older age, underlying pre-existing pathology, renal dysfunction, and concurrent use of non-steroidals or anticoagulants. ${ }^{25}$ Lanas et al showed that the relative risk for upper gastrointestinal bleeding was 3.7 for low-dose aspirin, 2.8 for clopidogrel and 16.4 for the combination of aspirin with clopidogrel. ${ }^{26}$ In the Clopidogrel in Unstable Angina to Prevent Recurrent Events (CURE) trial, aspirin plus clopidogrel combination prevented 23 new cardiovascular events in the place of 10 major bleeding episodes for every 1,000 patients; while in the case of prasugrel, the figures were 19 and 7, and for ticagrelor they were 22 and $6 .{ }^{12,14,25,27}$

It is preferable to prevent a bleed rather than treat it. Some simple measures may be followed in patients on antiplatelet therapy.

$>$ Regularly reassess the need for dual antiplatelets.

> Use a proton pump inhibitor (PPI), especially with dual antiplatelets or if prescribed with anticoagulant.

$>$ Treat Helicobacter pylori aggressively in the presence of any symptoms and positive microbiology.

$>$ It is preferable to avoid nonsteroidal anti-inflammatory drugs (NSAIDs) in patients receiving antiplatelet therapy although some patients may require them for musculoskeletal problems. Guidelines recommend that PPI therapy is used in such a context especially in older people and those at high risk of gastrointestinal bleeding. ${ }^{16}$ NSAIDs have also been suggested to antagonize the antiplatelet effects of aspirin. ${ }^{16}$

> Pharmacokinetics of aspirin can be influenced by the formulation and there are concerns whether low-dose enteric-coated aspirin is bioequivalent in efficacy to plain aspirin. It is possible that enteric coating decreases the antiplatelet effects and should be avoided. Studies done on volunteers suggest that enteric-coated aspirin preparations may only cause incomplete inhibition of the cyclo-oxygenase pathway compared with plain aspirin especially in heavier individuals which might lead to 'pseudoresistance. ${ }^{62}$

In a patient who developed a bleed:

$>$ investigate any bleed by endoscopy or other methods before attributing it to the antiplatelet agent as underlying pathology may have caused it

$>$ reduce the dose of single antiplatelet therapy if possible or switch to an agent with relatively less bleeding risk (aspirin to clopidogrel)

$>$ if bleeding occurred on dual therapy, reassess the need for continuation in discussion with a cardiologist

$>$ if dual therapy is a must, aspirin clopidogrel combination may be preferred.

Resumption of the antiplatelet is based on the thrombotic risk in the absence of restarting with continued bleeding risk. If a clear cause was not identifiable and the bleed developed despite being on PPI, a delay in starting is preferable. However, if a cause was found and appropriately treated and also there is high thrombotic risk (recent stent insertion), then early recommencement of the therapy should be considered. ${ }^{25}$

It is important to remember that intracranial bleeding can occasionally develop with antiplatelet drugs. Retroperitoneal haemorrhage is also a rare, overlooked complication of stent insertion in thin individuals while receiving antiplatelet drugs especially if they had a high femoral-artery puncture.

\section{Can proton pump inhibitors be used with antiplatelet drugs?}

Most guidelines recommend the use of PPIs in patients treated with antiplatelet therapy and, in particular, dual therapy, especially if there is a history of gastrointestinal bleed or peptic ulcer. However, there have been some reports which suggested that certain PPIs, especially omeprazole, might diminish the effects of antiplatelet drug, clopidogrel, through inhibition of CYP2C19. ${ }^{28}$ A systematic review showed concomitant PPI use might be associated with an increased risk of cardiovascular events in patients receiving clopidogrel but did not influence the risk of death. ${ }^{29}$ However, the COGENT (Clopidogrel and the Optimization of Gastrointestinal Events Trial) investigators who looked at clopidogrel with or without omeprazole in coronary artery disease did not show an increase in the risk of cardiovascular events. ${ }^{30}$ In the absence of clear and conclusive evidence, the expert position paper from the European Society of Cardiology recommends if PPIs are indicated, a formulation with less CYP2C19 inhibitory capacity (eg pantoprazole) should be chosen. ${ }^{31}$ There is however no restriction on the use of PPIs in patients on aspirin, prasugrel or ticagrelor.

\section{Dyspnoea with ticagrelor}

Dyspnoea is a very common ticagrelor side effect with an incidence of $10-20 \% .^{32}$ It is often described by patients as sudden and unexpected air hunger lasting for few minutes in most cases but occasionally much longer. ${ }^{32}$ They usually occur in the first week of treatment, and most episodes are reported as mild. The dyspnoea usually occurs at rest and is not associated with wheezing, or other symptoms of congestive heart failure or coronary ischaemia. ${ }^{32}$ The exact mechanism for the development of this complication is not known although pulmonary vagal nerve stimulation by the increased levels of adenosine or the inhibition of $\mathrm{P}_{2} \mathrm{Y}_{12}$ receptors on sensory neurons have been suggested as possibilities. ${ }^{33}$ Since the dyspnoea is mild in most patients, all that may be necessary is reassurance and counselling. However, if the dyspnoea is troublesome, consider switching to prasugrel (or clopidogrel if prasugrel is contraindicated) with a full loading dose 24 hours from the last ticagrelor dose. ${ }^{34}$

\section{Switching between $\mathrm{P}_{2} \mathrm{Y}_{12}$ inhibitors}

Dual antiplatelet therapy with aspirin and a $\mathrm{P}_{2} \mathrm{Y}_{12}$ inhibitor is standard after acute coronary syndromes, with either medical treatment or PCI. Occasionally one of the antiplatelet agents may require to be switched to a different kind. Some of the scenarios, where this is required are: elevated thrombotic risk while on treatment with one antiplatelet agent, when a more potent drug may be preferred and when high on-treatment platelet reactivity (HPR), a marker for the risk of ischaemic recurrences are proven while receiving aspirin or clopidogrel (resistance discussed later). ${ }^{35,36}$ 


\section{From clopidogrel to ticagrelor or prasugrel ${ }^{35-37}$}

$>$ In acute presentations, give $180 \mathrm{mg}$ loading dose of ticagrelor followed by $90 \mathrm{mg}$ bd maintenance dose 12 hours after disregarding the timing of the last clopidogrel dose.

> In patients with stable clinical presentations, the loading dose is avoided and the drug is initiated when the next dose of clopidogrel would be due ( 24 hour from the last dose of clopidogrel).

$>$ In the case of prasugrel, a similar method is followed with $60 \mathrm{mg}$ loading dose followed by $10 \mathrm{mg}$ maintenance dose in the acute setting, or $10 \mathrm{mg}$ maintenance dose started in the stable patients when the next dose of clopidogrel is due.

\section{Ticagrelor or prasugrel to clopidogrel ${ }^{35}$}

$>$ Clopidogrel should be started at a loading dose of 300$600 \mathrm{mg} 24$ hour after the last maintenance dose of ticagrelor; the loading dose is given because the antiplatelet action of ticagrelor is short.

$>$ Clopidogrel can be started at $75 \mathrm{mg}, 24$ hours after the last dose of prasugrel although some experts recommend loading dose if the switch is done in a high thrombotic setting.

\section{Between ticagrelor and clopidogrel ${ }^{35,38}$}

$>$ A need for a switch between prasugrel and ticagrelor is rare but may be needed for compliance issues since ticagrelor is a twice daily drug and prasugrel is taken once a day.

$>$ Patients who develop troublesome dyspnoea with ticagrelor may be better off on prasugrel.

$>$ Reduction in body mass or development of a stroke during prasugrel treatment.

> A loading dose of $60 \mathrm{mg}$ of prasugrel is given 24 hours after the last dose of ticagrelor, based on the Switching Anti Platelet-2 study (SWAP-2), while a similar method of loading dose of ticagrelor after prasugrel is being investigated in the SWAP-3 study.

\section{Triple therapy (Fig 1)}

Anticoagulant therapy has established its place in reducing systemic thrombotic events in patients with atrial fibrillation.
The heart rhythm problem is likely to also be common in patients who develop ischaemic events and require antiplatelet therapy. In such patients who may require both antiplatelet and anticoagulant agent, despite the clear benefits from preventing thrombotic episodes, it can also lead to unacceptable bleeding. Certain general principles should be followed in this setting, which are based on the recent guidelines and consensus. ${ }^{39,40}$

> Triple therapy with aspirin and clopidogrel in combination with an anticoagulant like warfarin or a direct oral anticoagulant should be given in patients who have had a stent inserted at least in the initial stages.

$>$ Depending on the type of stent and the risk of bleeding, the duration of triple therapy should be kept to a minimum (one or three or six months).

> After 6-12 months from a stent insertion or acute coronary syndrome the risk of stent thrombosis or recurrent cardiac events is reduced. Therefore the antiplatelet agents should be discontinued and anticoagulation should be continued long term.

> In the setting of PCI, a bare metal stent or one of the newer drug-eluting stents with lower thrombotic risk should be preferentially utilised to minimise the duration of triple therapy.

$>$ If warfarin is the anticoagulant chosen, the target international normalised ratio (INR) should be kept between 2.0 and 2.5 (if appropriate) until the dual antiplatelet agents are continued.

> The newer $\mathrm{P}_{2} \mathrm{Y}_{12}$-receptor inhibitors, including ticagrelor and prasugrel, should not be used currently as part of triple therapy until further data are available.

$>$ Stomach protection with PPIs should be considered in all patients on triple therapy.

If the patient on antiplatelet therapy develops atrial fibrillation, the choice of oral anticoagulant therapy to be a part of the triple therapy is based on the same considerations as when the individual is not on antiplatelets ie dependent on the $\mathrm{CHADS}_{2}$-VASC and HAS BLED scores. ${ }^{41}$ If the bleeding risk is high, warfarin with a target INR of 2.0-2.5, apixaban at a lower dose of $2.5 \mathrm{mg}$ bd or rivaroxaban $15 \mathrm{mg}$ od should be chosen. ${ }^{41}$ Outside the setting of coronary disease, addition of an antiplatelet agent to anticoagulation does not have a good risk-benefit ratio except for in patients with a mechanical heart

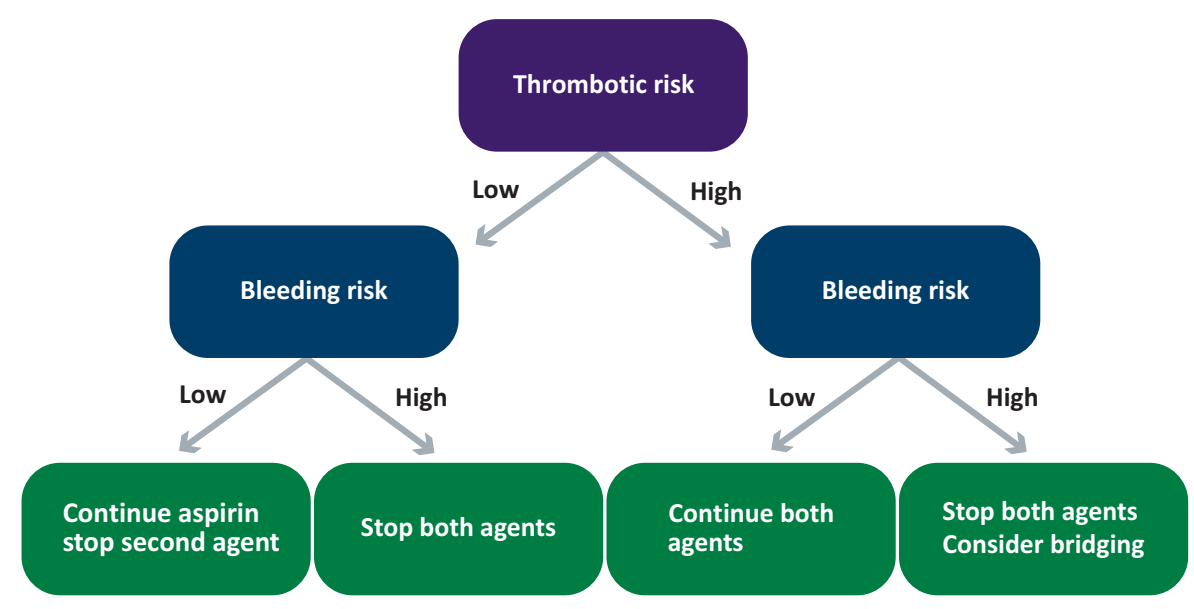

Fig 1. A practical algorithm for the management of procedures in patients receiving dual antiplatelet therapy. The dual agents are usually aspirin and a $\mathrm{P}_{12} \mathrm{Y}_{12}$ inhibitor. Bridging is with a glycoprotein IIb/IIIa blocker. 
valve, especially if they developed a systemic embolism despite therapeutic INR or ischaemic heart disease. ${ }^{42}$

\section{Thrombocytopenia and antiplatelet drugs}

Although not evidence-based most experts would recommend a platelet count of $50 \times 10^{9} / \mathrm{L}$ as a threshold for using antiplatelet drugs. This would mean patients with new-onset thrombocytopenia would need frequent blood checks to ensure the counts remain above the threshold to continue the antiplatelet agent. In all such cases, an active search for the cause of thrombocytopenia should be sought if necessary with assistance from a haematologist. It is useful to bear in mind that a common cause of isolated thrombocytopenia is a recent infection or the commencement of a new drug (to be discontinued). ${ }^{43}$ In both these cases, the thrombocytopenia may be transient and may allow resumption of antiplatelet therapy after an interval. Antiphospholipid syndrome is another condition which may present as an arterial event especially in younger individuals (necessitating the antiplatelet therapy) and may present with thrombocytopenia at a later stage. ${ }^{44}$ An extremely rare complication of clopidogrel is the development of thrombotic thrombocytopenic purpura which manifests with haemolytic anaemia and low platelet count; however this association has been disputed. ${ }^{45}$

\section{Interventional or surgical procedures while on antiplatelet agents}

Patients who are receiving antiplatelet therapy as a single agent or dual therapy are likely to need procedures. These may be interventional, for example endoscopies or surgical - both of which may be associated with an increased risk of bleeding. The bleeding risk in these cases is dependent on various factors including the site and invasiveness. A clear management policy is required in such patients after balancing the risks and benefits of continuation or discontinuation of the antiplatelet drugs. This plan should be made as a team involving the cardiologist, transfusion specialists and the proceduralist. A general guide follows (also see Fig 2). ${ }^{46,47}$

$>$ An assessment of the thrombotic risk of the patient is considered first. Clarification about the necessity for the prescription of antiplatelet therapy and, the likely thrombotic risk from stopping the drug before the procedure. A recent ischaemic event (myocardial infarction or cerebrovascular event in the previous 4 months) is probably the best indicator of high thrombotic risk. In the context of percutaneous angioplasty, the first 6 months after a drug-eluting or bare metal stent is considered as high thrombotic risk although some drug-eluting stents (first generation) may have a risk up to 12 months. Premature discontinuation of antiplatelet therapy in these cases is associated with a very high risk of stent thrombosis and mortality.

$>$ Next the bleeding risk of the procedure is ascertained. This is done by the operator but a procedure which requires transfusions even in the absence of antiplatelet therapy is usually considered as high risk of bleeding. In addition, if the surgery is being undertaken in a closed space like intracranial or posterior chamber of the eye, they are considered high risk of bleeding.

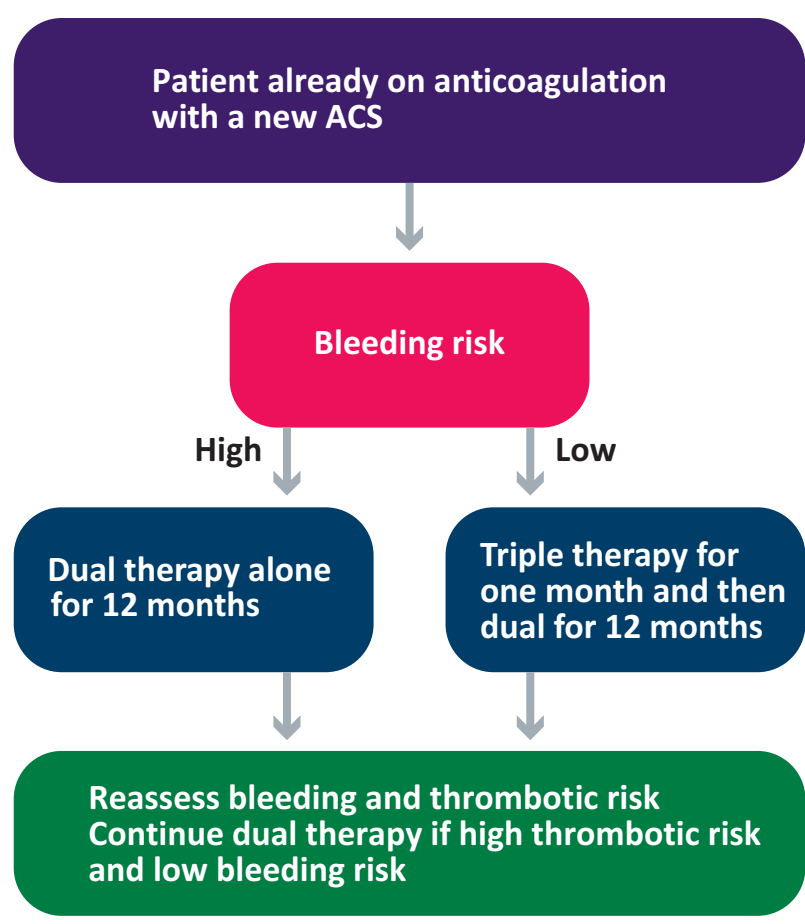

Fig 2. Algorithm for the management of triple therapy including the duration.

$>$ If the bleeding risk is low and thrombotic risk is high, it is probably worth continuing the single or dual antiplatelet therapy (especially if a coronary stent was inserted in the preceding 6-12 months).

$>$ If the bleeding risk is high, all antiplatelet agents should be stopped. If at all possible, the procedure should be undertaken after the stipulated time period (where the need for antiplatelet therapy is high) has elapsed. If discontinuation is planned, current guidelines recommend stopping clopidogrel and ticagrelor 5 days before and prasugrel 7 days before the procedure. ${ }^{46,47}$

$>$ In the case of emergency procedures, a discussion is mandatory between the cardiologist and person undertaking the procedure. Current practice is based on platelet transfusions which may be given to 'overcome' the effect of the antiplatelet agents. There are some data suggesting that this approach may be helpful in the case of aspirin but not clopidogrel (probably due to plasma half-life differences). ${ }^{47,48}$ There is also the theoretical risk of stent thrombosis with platelet transfusions.

> In relation to regional anaesthesia and spinal procedures, the American and European guidelines suggest neuraxial and peripheral blocks can be safely performed while on aspirin but not on the $\mathrm{P}_{2} \mathrm{Y}_{12}$ blockers. ${ }^{49,50}$ An interval of 7-10 days for clopidogrel and prasugrel, and 5 days for ticagrelor is recommended.

\section{The conundrum of antiplatelet resistance}

Aspirin and clopidogrel are very effective drugs in preventing cardiovascular ischaemia in patients with 
coronary artery or cerebrovascular disease. However, there have been occasional cases of recurrent thrombosis, especially stent thrombosis, despite being treated with these agents. This indicates the possibility of 'antiplatelet resistance', particularly if the patient has been compliant with the drugs. ${ }^{51}$ The common reasons quoted for aspirin and clopidogrel resistance are: ${ }^{51,52}$

$>$ non-compliance

$>$ genetic polymorphisms (COX-1 polymorphisms for aspirin and CYP450 isoenzymes for clopidogrel)

$>$ smoking (smoker's paradox), diabetes and hypercholesterolemia which can enhance platelet function

$>$ higher body mass index enhancing platelet reactivity

> variable clopidogrel metabolite generation due to altered limited intestinal absorption

$>$ co-administration of PPIs such as omeprazole and clopidogrel.

Although measurement of aspirin resistance is not often performed for want of an alternative COX inhibitor, measurement of clopidogrel resistance is occasionally considered due to the availability of more potent agents in the same class (albeit at higher cost). Clopidogrel resistance is identified by determining the high on-treatment platelet reactivity (HPR) during treatment (not by measuring the difference in platelet inhibition before and after therapy). Although in the setting of patients with coronary artery disease undergoing PCI, an increased HPR is a risk of stent thrombosis; in the non-interventional, stable patients, the relevance of HPR is uncertain. Based on the results, guidelines suggest platelet function testing to be considered in patients at high risk for poor clinical outcomes, and those with HPR on clopidogrel, alternative agents, such as prasugrel or ticagrelor, might be considered. ${ }^{53}$ They discourage routine use of these tests for screening. Some recent reports hint at an early HPR (in the first 4 hours) in patients receiving prasugrel and ticagrelor after undergoing PCI currently attributed to the concomitant use of morphine but further studies are required for confirmation. ${ }^{51}$

In a similar way to HPR as a risk factor for recurrent ischaemic events, low platelet responsiveness has been suggested to be a risk factor for bleeding in patients on aspirin or clopidogrel. ${ }^{51}$ The multicentre, platelet reactivity and clinical outcomes after coronary artery implantation of drug-eluting stents (ADAPT-DES) study showed the problems of both HPR and low platelet responsiveness in causing thrombosis and bleeding respectively. ${ }^{54}$ The Society of Thoracic Surgeons now recommend the use of platelet function testing to assist the timing of surgery in patients treated with clopidogrel. ${ }^{55}$

The commonest argument against routine use of HPR is the fact that it is not the only predictor of recurrent thrombosis. Attempts at including clinical markers and genotype to HPR may prove helpful in this regard. ${ }^{10,51}$ The concept of a 'therapeutic window' based on HPR leading to personalised antiplatelet therapy aimed at maximum thrombosis prevention with minimal bleeding is very attractive but validation is required. ${ }^{10,51}$

\section{Aspirin and cancer}

In recent years, there has been considerable interest in the role of aspirin as an anticancer agent. An analysis of all randomised trials of daily aspirin versus no aspirin for at least 4 years noted the 20-year risk of cancer death was significantly lower for all solid cancers and gastrointestinal cancers. ${ }^{56}$ The benefit was unrelated to aspirin dose but did increase with age and also the duration of treatment. ${ }^{56}$ The strongest evidence in this area is in the prevention of colon cancer with daily aspirin being associated with a $20 \%$ reduced risk; although its use following the diagnosis of the colorectal cancer does not improve the overall survival rate ${ }^{57,58}$ Despite this remarkable effect, there is still lack of evidence in what is the best dose of aspirin with anticancer properties. The suggested mechanisms for this favourable effect include induction of apoptosis, inhibition of angiogenesis and antiproliferative effects mediated via COX-dependent and -independent pathways. ${ }^{57-59}$ Aspirin may also have a role in preventing metastasis through its antiplatelet effect. ${ }^{59}$ Tumour cells induce platelet aggregation as a mechanism to evade immune surveillance and thus facilitate their metastasis, which is blocked by aspirin. ${ }^{59,60}$ From a practical point of view, a net benefit analysis discussion should be made with the patient about the need for long-term use of aspirin necessary to prevent the cancer and the increased risk of bleeding associated with it.

\section{Conclusion}

The antiplatelet drug therapy landscape has considerably changed in the last decade with the arrival of several new drugs with different mechanisms of action. This has provided us with more choices for their use in the different settings with the ability to balance bleeding and thrombotic risks. More potent drugs have certainly had an impact in reducing the thrombotic complications in cardiovascular diseases, especially in the PCI setting. It is useful to know the several uncommon but definite adverse effects these agents may have and also the appropriate situations for their use. The very interesting anticancer role for aspirin indicates that the modulation of platelet function may have many more benefits than just the prevention of thrombosis.

\section{References}

1 Patrono C. Aspirin as an antiplatelet drug. N Engl J Med 1994;330:1287-94.

2 Campbell CL, Smyth S, Montalescot G, Steinhubl SR. Aspirin dose for the prevention of cardiovascular disease: a systematic review. JAMA 2007;297:2018-24.

3 Angiolillo DJ. The evolution of antiplatelet therapy in the treatment of acute coronary syndromes: from aspirin to the present day. Drugs 2012;72:2087-116.

4 Franchi F, Angiolillo DJ. Novel antiplatelet agents in acute coronary syndrome. Nat Rev Cardiol 2015;12:30-4.

5 Gresele P, Momi S, Falcinelli E. Anti-platelet therapy: phosphodiesterase inhibitors. Br J Clin Pharmacol 2011;72:634-46

6 Eisert WG. Dipyridamole in antithrombotic treatment. Adv Cardiol 2012;47:78-86.

7 ESPRIT Study Group. Aspirin plus dipyridamole versus aspirin alone after cerebral ischaemia of arterial origin (ESPRIT): randomised controlled trial. Lancet 2006;367:1665-73.

8 Rogers KC, Oliphant CS, Finks SW. Clinical efficacy and safety of cilostazol: a critical review of the literature. Drugs 2015;75:377-95.

9 Alonso-Coello P, Bellmunt S, McGorrian C et al. Antithrombotic therapy in peripheral artery disease: antithrombotic therapy and prevention of thrombosis, 9th ed: American College of Chest 
Physicians evidence-based clinical practice guidelines. Chest 2012;141(2 Suppl):e669S-90S.

10 Wiviott SD, Steg PG. Clinical evidence for oral antiplatelet therapy in acute coronary syndromes. Lancet 2015;386:292-302

11 Wallentin L, Varenhorst C, James S et al. Prasugrel achieves greater and faster P2Y12receptor-mediated platelet inhibition than clopidogrel due to more efficient generation of its active metabolite in aspirin-treated patients with coronary artery disease. Eur Heart $J$ 2008;29:21-30.

12 Wiviott SD, Braunwald E, McCabe CH et al. TRITON-TIMI 38 Investigators. Prasugrel versus clopidogrel in patients with acute coronary syndromes. N Eng J Med 2007;357:2001-15.

13 Montalescot G, Wiviott SD, Braunwald E et al. Prasugrel compared with clopidogrel in patients undergoing percutaneous coronary intervention for ST-elevation myocardial infarction (TRITONTIMI 38): double-blind, randomised controlled trial. Lancet 2009:373:723-31.

14 Wallentin L, Becker RC, Budaj A et al. Ticagrelor versus clopidogrel in patients with acute coronary syndromes. $\mathrm{N} \mathrm{Engl} \mathrm{J} \mathrm{Med}$ 2009;361:1045-57.

15 Cannon CP, Harrington RA, James S et al. Comparison of ticagrelor with clopidogrel in patients with a planned invasive strategy for acute coronary syndromes (PLATO): a randomised doubleblind study. Lancet 2010;375:283-93.

16 National Institute for Health and Care Excellence. Antiplatelet treatment. London: NICE, 2015. Available online at http://cks.nice.org. uk/antiplatelet-treatment [Accessed 16 February 2016].

17 Topol E, Byzova T, Plow E. Platelet GP IIb-IIIa blockers. Lancet 1999;353:227-31

18 Muñiz-Lozano A, Rollini F, Franchi F et al. Update on platelet glycoprotein IIb/IIIa inhibitors: recommendations for clinical practice. Ther Adv Cardiovasc Dis 2013;7:197-213.

19 Savonitto S, D'Urbano M, Caracciolo M et al. Urgent surgery in patients with a recently implanted coronary drug-eluting stent: a phase II study of 'bridging' antiplatelet therapy with tirofiban during temporary withdrawal of clopidogrel. Br J Anaesth 2010;104:285-91.

20 Steg PG. Bhatt DL, Hamm CW et al. Effect of cangrelor on periprocedural outcomes in percutaneous coronary interventions: a pooled analysis of patient-level data. Lancet 2013;382;1981-92.

21 Angiolillo DJ, Firstenberg MS, Price MJ et al. Bridging antiplatelet therapy with cangrelor in patients undergoing cardiac surgery: a randomized controlled trial. JAMA 2012;307:265-74.

22 Morrow DA, Braunwald E, Bonaca MP et al. Vorapaxar for secondary prevention of atherothrombotic events. $N$ Engl J Med 2012;366:1404-13.

23 Amsterdam EA, Wenger NK, Brindis RG et al. 2014 AHA/ACC Guideline for the Management of Patients With Non-ST-Elevation Acute Coronary Syndromes: a report of the American College of Cardiology/American Heart Association Task Force on Practice Guidelines. Circulation 2014;130:e344-426.

24 Antithrombotic Trialists' (ATT) Collaboration. Aspirin in the primary and secondary prevention of vascular disease: collaborative meta-analysis of individual participant data from randomised trials. Lancet 2009;373:1849-60.

25 Pipilis A, Makrygiannis S, Chrisanthopoulou E et al. Gastrointestinal bleeding in patients receiving antiplatelet and anticoagulant therapy: practical guidance for restarting therapy and avoiding recurrences. Hellenic J Cardiol 2014;55:499-509.

26 Lanas A, García-Rodríguez LA, Arroyo MT et al. Risk of upper gastrointestinal ulcer bleeding associated with selective cyclo-oxygenase-2 inhibitors, traditional non-aspirin nonsteroidal anti-inflammatory drugs, aspirin and combinations. Gut 2006;55:1731-8.

27 Yusuf S, Zhao F, Mehta SR et al; Clopidogrel in Unstable Angina to Prevent Recurrent Events Trial Investigators. Effects of clopidogrel in addition to aspirin in patients with acute coro- nary syndromes without ST-segment elevation. N Engl J Med 2001;345:494-502.

28 Gilard M, Arnaud B, Cornily JC et al. Influence of omeprazole on the antiplatelet action of clopidogrel associated with aspirin: the randomized, double-blind OCLA (Omeprazole CLopidogrel Aspirin) study. J Am Coll Cardiol 2008;51:256-60.

29 Siller-Matula JM, Jilma B, Schrör K et al. Effect of proton pump inhibitors on clinical outcome in patients treated with clopidogrel: a systematic review and meta-analysis. J Thromb Haemost 2010;8:2624-41.

30 Bhatt DL, Cryer BL, Contant CF et al. COGENT Investigators. Clopidogrel with or without Omeprazole in Coronary Artery Disease. N Engl J Med 2010;363:1909-17.

31 Agewall S, Cattaneo M, Collet JP et al; ESC Working Group on Cardiovascular Pharmacology and Drug Therapy and ESC Working Group on Thrombosis. Expert position paper on the use of proton pump inhibitors in patients with cardiovascular disease and antithrombotic therapy. Eur Heart J 2013;34:1708-13,1713a-1713b.

32 Storey RF, Becker RC, Harrington RA et al. Characterization of dyspnoea in PLATO study patients treated with ticagrelor or clopidogrel and its association with clinical outcomes. Eur Heart $J$ 2011;32:2945-53.

33 Catteneo M, Faioni EM. Why does ticagrelor induce dyspnea? Thromb Haemost 2012;108:1031-6.

34 Parodi G, Storey RF. Dyspnoea management in acute coronary syndrome patients treated with ticagrelor. Eur Heart J 2015;4:555-60.

35 Rollini F, Franchi F, Angiolillo DJ. Switching P2Y12-receptor inhibitors in patients with coronary artery disease. Nat Rev Cardiol 2016;13:11-2.

36 Angiolillo DJ, Saucedo JF, Deraad R et al. Increased platelet inhibition after switching from maintenance clopidogrel to prasugrel in patients with acute coronary syndromes: results of the SWAP (SWitching Anti Platelet) study. J Am Col Cardiol 2010;56:1017-23.

37 Gurbel PA, Bliden KP, Butler K, et al. Response to ticagrelor in clopidogrel nonresponders and responders and effect of switching therapies: the RESPOND study. Circulation 2010;121:1188-99.

38 Angiolillo DJ, Curzen N, Gurbel P et al. Pharmacodynamic evaluation of switching from ticagrelor to prasugrel in patients with stable coronary artery disease: results of the SWAP-2 study (Switching Anti Platelet-2). J Am Col Cardiol 2014;63:1500-9.

39 Lip GY, Windecker S, Huber K et al. Management of antithrombotic therapy in atrial fibrillation patients presenting with acute coronary syndrome and/or undergoing percutaneous coronary or valve interventions: a joint consensus document of the EHRA, EAPCI and ACCA endorsed by the Heart Rhythm Society and Asia-Pacific Heart Rhythm Society (APHRS). Eur Heart J 2014;35:3155-79.

40 Rubboli A, Faxon DP, Juhani Airaksinen KE et al. The optimal management of patients on oral anticoagulation undergoing coronary artery stenting. The 10th anniversary overview, Thromb Haemost 2014;112:1080-7.

41 Rubboli A, Agewall S, Huber K, Lip GY. New-onset atrial fibrillation after recent coronary stenting: Warfarin or non-vitamin $\mathrm{K}$-antagonist oral anticoagulants to be added to aspirin and clopidogrel? A viewpoint. Int J Cardiol 2015;196:133-8.

42 Dentali F, Douketis JD, Lim W et al. Combined aspirin-oral anticoagulant therapy compared with oral anticoagulant therapy alone among patients at risk for cardiovascular disease: a meta-analysis of randomized trials. Arch Intern Med 2007;167:117-24.

43 Thachil J, Fitzmaurice D. Thrombocytopenia in an adult. BMJ 2013;346:f3407.

44 Ruiz-Irastorza G, Crowther M, Branch W et al. Antiphospholipid syndrome. Lancet 2010;376:1498-509.

45 Bennett CL, Connors JM, Carwile JM et al. Thrombotic thrombocytopenic purpura associated with clopidogrel. N Engl J Med 2000;342:1773-7.

46 Douketis JD, Spyropoulos AC, Spencer FA et al. Perioperative management of antithrombotic therapy: Antithrombotic Therapy 
and Prevention of Thrombosis, 9th ed: American College of Chest Physicians Evidence-Based Clinical Practice Guidelines. Chest 2012;141:e326-50S.

47 Oprea AD, Popescu WM. Perioperative management of antiplatelet therapy. Br J Anaesth 2013;111 Suppl 1:i3-17.

48 Taylor G, Osinski D, Thevenin A et al. Is platelet transfusion efficient to restore platelet reactivity in patients who are responders to aspirin and/or clopidogrel before emergency surgery? J Trauma Acute Care Surg 2013;74:1367-9.

49 Horlocker TT, Wedel DJ, Rowlingson JC et al. Regional anesthesia in the patient receiving antithrombotic or thrombolytic therapy: American Society of Regional Anesthesia and Pain Medicine Evidence-Based Guidelines (Third Edition). Reg Anesth Pain Med 2010;35:64-101.

50 Gogarten W, Vandermeulen E, Van Aken H et al. Regional anaesthesia and antithrombotic agents: recommendations of the European Society of Anaesthesiology. Eur J Anaesthesiol 2010;27:999-1015.

51 Tantry US, Gesheff M, Liu F, Bliden KP, Gurbel PA. Resistance to antiplatelet drugs: what progress has been made? Expert Opin Pharmacother 2014;15:2553-64.

52 Tantry US, Bonello L, Aradi D et al. Working Group on On-Treatment Platelet Reactivity. Consensus and update on the definition of on-treatment platelet reactivity to adenosine diphosphate associated with ischemia and bleeding. J Am Coll Cardiol 2013;62:2261-73.

53 Levine GN, Bates ER, Blankenship JC et al. 2011. ACCF/AHA/SCAI Guideline for Percutaneous Coronary Intervention: a report of the American College of Cardiology Foundation/American Heart Association Task Force on Practice Guidelines and the Society for Cardiovascular Angiography and Interventions. J Am Coll Cardiol 2011;58:e44-122.

54 Stone GW, Witzenbichler B, Weisz G, et al; ADAPT-DES Investigators. Platelet reactivity and clinical outcomes after coro- nary artery implantation of drug-eluting stents (ADAPT-DES): a prospective multicentre registry study. Lancet. 2013;382:614-23.

55 Ferraris VA, Saha SP, Oestreich JH et al. Society of Thoracic Surgeons. 2012 update to the Society of Thoracic Surgeons guideline on use of antiplatelet drugs in patients having cardiac and noncardiac operations. Ann Thorac Surg 2012;94:1761-81.

56 Rothwell PM, Fowkes FG, Belch JF et al. Effect of daily aspirin on long-term risk of death due to cancer: analysis of individual patient data from randomised trials. Lancet 2011;377:31-41.

57 Ye X, Fu J, Yang Y et al. Dose-risk and duration-risk relationships between aspirin and colorectal cancer: a meta-analysis of published cohort studies. PLoS One 2013;8:e57578.

58 Cardwell CR, Kunzmann AT, Cantwell MM et al. Low-dose aspirin use after diagnosis of colorectal cancer does not increase survival: a case-control analysis of a population-based cohort. Gastroenterology 2014;146:700-8.

59 Usman MW, Luo F, Cheng $\mathrm{H}$ et al. Chemopreventive effects of aspirin at alance. Biochim Biophys Acta 2015;1855:254-63.

60 Gay LJ, Felding-Habermann B, Contribution of platelets to tumour metastasis, Nat Rev Cancer 2011;2011:123-34.

61 Franchi F, Angiolillo DJ. Novel antiplatelet agents in acute coronary syndrome. Nat Rev Cardiol 2015;12:30-4.

62 Clark MG, Beavers C, Osborne J. Managing the acute coronary syndrome patient: Evidence based recommendations for anti-platelet therapy. Heart Lung 2015;44:141-9.

Address for correspondence: Dr J Thachil, Department of Haematology, Central Manchester University Hospitals NHS Foundation Trust, Oxford Road, Manchester M13 9WL, UK. Email: jecko.thachil@cmft.nhs.uk

\section{.

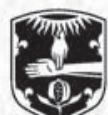 \\ Royal College of Physicians}

\section{Gifts and memorabilia}

A new range of gifts inspired by our latest RCP exhibition: Scholar, courtier, magician: the lost library of John Dee (18 January - 29 July 2016).

Postcards $£ 2.50$ for six (contains one of each design)

Order by phone or online $\mathrm{Tel}+44(0) 2030751358$ or email publications@rcplondon.ac.uk

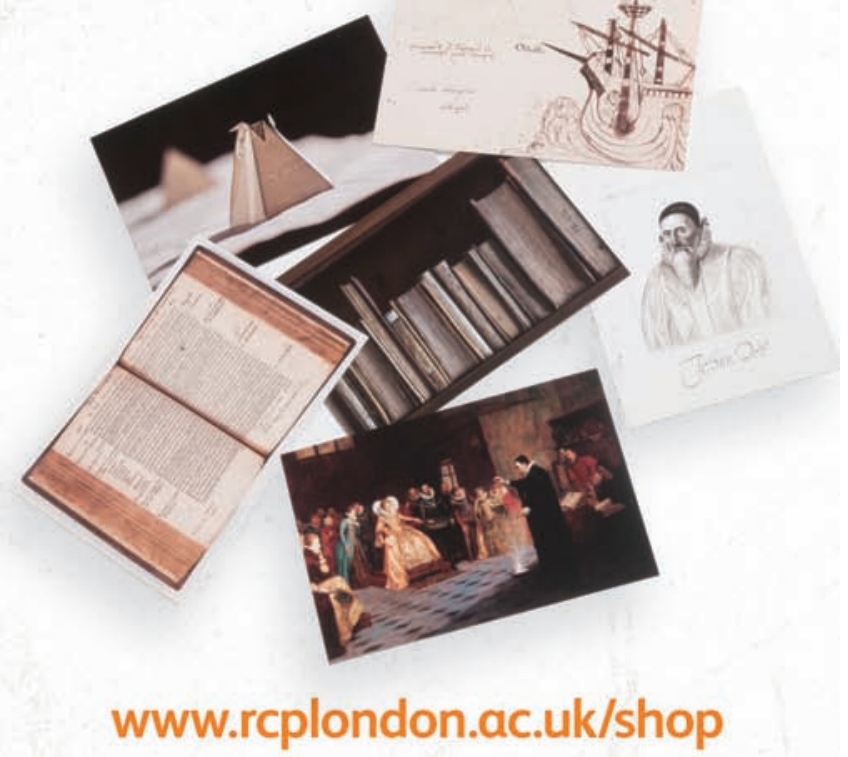

